\title{
Fixed point algorithms for the split problem of demicontractive operators
}

\author{
Xiaoxue Zheng ${ }^{\mathrm{a}}$, Yonghong Yao ${ }^{\mathrm{a}}$, Yeong-Cheng Liou ${ }^{\mathrm{b}, *}$, Limin Leng ${ }^{\mathrm{a}}$ \\ ${ }^{a}$ Department of Mathematics, Tianjin Polytechnic University, Tianjin 300387, China. \\ ${ }^{b}$ Department of Healthcare Administration and Medical Informatics, and Research Center of Nonlinear Analysis and Optimization, \\ Kaohsiung Medical University, and Department of Medical Research, Kaohsiung Medical University Hospital, Kaohsiung 807, Taiwan.
}

Communicated by N. Shahzad

\begin{abstract}
A fixed point method is introduced for solving the split common fixed point problem of demicontractive operators in Hilbert spaces. By virtue of this fixed point method, we construct an iteration based on Mann's method for solving the split common fixed point problem of demicontractive operators. Weak convergence analysis is given under some mild assumptions. (C)2017 All rights reserved.
\end{abstract}

Keywords: Split common fixed point, demicontractive operator, weak convergence. 2010 MSC: 47H10, 65J15.

\section{Introduction}

The main purpose of the present paper is to study the split common fixed point problem. To begin with, let us state the related background.

Throughout, $\mathrm{H}_{1}$ and $\mathrm{H}_{2}$ are two real Hilbert spaces, $\langle\cdot, \cdot\rangle$ denotes the inner product, and $\|\cdot\|$ stands for the corresponding norm. Let $\mathrm{U}: \mathrm{H}_{1} \rightarrow \mathrm{H}_{1}$ and $\mathrm{T}: \mathrm{H}_{2} \rightarrow \mathrm{H}_{2}$ be two nonlinear operators. We use Fix $(\mathrm{U})$ and $\operatorname{Fix}(T)$ to denote the fixed point sets of $U$ and $T$, respectively. Let $A: H_{1} \rightarrow H_{2}$ be a bounded linear operator with its adjoint $A^{*}$.

Recall that the split common fixed point problem is to seek an element $x^{*} \in \mathrm{H}_{1}$ such that

$$
x^{*} \in \operatorname{Fix}(U) \text { and } A x^{*} \in \operatorname{Fix}(T) .
$$

Remark 1.1. The split common fixed point problem (1.1) is a generalization of the split feasibility problem arising from signal processing and image restoration $([3,4,10,13,14,16,20])$, which is to find a point $x^{*}$ such that

$$
x^{*} \in C \text { and } A x^{*} \in Q \text {, }
$$

where $\mathrm{C} \subset \mathrm{H}_{1}$ and $\mathrm{Q} \subset \mathrm{H}_{2}$ are two nonempty closed convex sets.

\footnotetext{
${ }^{*}$ Corresponding author

Email addresses: zhengxiaoxue1991@aliyun.com (Xiaoxue Zheng), yaoyonghong@aliyun.com (Yonghong Yao), simplex_liou@hotmail.com (Yeong-Cheng Liou), lenglimin@aliyun.com (Limin Leng)

doi:10.22436/jnsa.010.03.35
} 
Problem (1.1) was firstly introduced by Censor and Segal [5]. Note that, solving (1.1) can be translated to solve the fixed point equation

$$
x^{*}=\mathrm{U}\left(x^{*}-\tau A^{*}(\mathrm{I}-\mathrm{T}) A x^{*}\right), \tau>0 .
$$

Whereafter, Censor and Segal proposed the following algorithm for directed operators.

Algorithm 1.2. Initialization: let $\chi^{*} \in \mathrm{H}_{1}:=\mathbb{R}^{\mathfrak{n}}$ be arbitrary.

Iterative step: for $n \geqslant 0$, let

$$
x_{n+1}=U\left(x_{n}-\tau A^{*}(I-T) A x_{n}\right), \quad n \geqslant 0,
$$

where $U: \mathbb{R}^{n} \rightarrow \mathbb{R}^{n}$ and $T: \mathbb{R}^{m} \rightarrow \mathbb{R}^{m}$ are two directed operators and $\tau \in(0,2 / \lambda)$ with $\lambda$ being the spectral radius of the operator $A^{*} A$.

Furthermore, Moudafi [8] considered the following relaxation version of (1.3) for k-demicontractive operators.

Algorithm 1.3. Initialization: let $\mathrm{x}_{0} \in \mathrm{H}_{1}$ be arbitrary.

Iterative step: for $n \geqslant 1$, assume the $n$-th iteration $x_{n}$ is constructed. Compute the $(n+1)$-th iteration $x_{n+1}$ by the following form

$$
\left\{\begin{array}{l}
y_{n}=x_{n}-\tau A^{*}(I-T) A x_{n}, \\
x_{n+1}=\left(1-\alpha_{n}\right) y_{n}+\alpha_{n} u\left(y_{n}\right), \quad n \geqslant 1,
\end{array}\right.
$$

where $\alpha_{n} \in(0,1)$ and $\tau \in\left(0, \frac{1-k}{\gamma}\right)$ with $\gamma$ being the spectral radius of the operator $A^{*} A$.

Since then, there has been growing interest in the split common fixed point problem $[6,14,19]$. In particular, Wang [9] noted that problem (1.1) can be cast as solving the following fixed-point equation

$$
x=x-\tau\left[(x-U x)+A^{*}(I-T) A x\right],
$$

where $\tau>0$ is a constant and $\mathrm{U}$ and $\mathrm{T}$ are directed operators.

Based on the equation (1.4), Wang [9] suggested the following iterative scheme:

$$
x_{n+1}=x_{n}-\tau\left[\left(x_{n}-U x_{n}\right)+A^{*}(I-T) A x_{n}\right], n \geqslant 0,
$$

where the stepsize $\tau$ is in the interval $\left(0, \frac{1}{\max \left\{1,\|A\|^{2}\right\}}\right)$ and proved weak convergence of the sequence $\left\{x_{n}\right\}$ to the solution of problem (1.1).

Very recently, Yao et al. [17] further suggested another new fixed point equation

$$
x=U x-\tau A^{*}(I-T) A x,
$$

where $\tau>0$ is a constant and $\mathrm{U}$ and $\mathrm{T}$ are directed operators.

Remark 1.4. Fixed point equation (1.5) is different from (1.4). Fixed point equations (1.4) and (1.5) rely on the properties of the operators $\mathrm{U}$ and $\mathrm{T}$.

On the other hand, construction of fixed points of nonlinear operators is an important and active research area. Iterative methods for finding fixed points of nonexpansive mappings have received vast investigation by Browder and Petryshyn [2], Wittmann [11], Xu [12], Yao et al. [15, 18, 21], and Zegeye et al. [22]. An important way for finding fixed points of nonexpansive mappings is Mann's method which generates a sequence $\left\{x_{n}\right\}$ by the form

$$
x_{n+1}=\left(1-\alpha_{n}\right) x_{n}+\alpha_{n} T x_{n}, n \geqslant 0,
$$

where $\alpha_{n} \in(0,1)$.

Inspired by the work in the literature, the main purpose of this paper is to apply the fixed point method for solving the split common fixed point problem (1.1). We first extend fixed point equation from the directed operators to the demicontractive operators. Subsequently, we construct an iteration based on Mann's method for solving the split common fixed point problem. Weak convergence theorem is given under some mild assumptions. 


\section{Preliminaries}

Let $\mathrm{C}$ be a nonempty closed convex subset of a real Hilbert space $\mathrm{H}$.

Definition 2.1. An operator $T: C \rightarrow C$ is said to be directed if

$$
\left\|T x-x^{\dagger}\right\|^{2} \leqslant\left\|x-x^{\dagger}\right\|^{2}-\|T x-x\|^{2}
$$

for all $x \in C$ and $x^{\dagger} \in \operatorname{Fix}(T)$.

The class of directed operators is an important class since it includes the orthogonal projections and the subgradient projectors which are fundamental in the convex optimization.

Definition 2.2. An operator $T$ is called demicontractive if there exists a constant $\beta \in[0,1)$ such that

$$
\|\mathrm{T} x-\mathrm{q}\|^{2} \leqslant\|x-\mathrm{q}\|^{2}+\beta\|x-\mathrm{T} x\|^{2},
$$

or equivalently

$$
\langle x-T x, x-q\rangle \geqslant \frac{1-\beta}{2}\|x-T x\|^{2}
$$

for all $(x, q) \in H \times \operatorname{Fix}(T)$.

Remark 2.3. It is clear that the demicontractive operators include the directed operators as special cases. The class of demicontractive operators is fundamental because many common types of operators arising in optimization belong to this class, see for example [7] and references therein.

Definition 2.4. An operator $T$ is said to be demiclosed if for any sequence $\left\{x_{n}\right\}$ which weakly converges to $\tilde{x}$, and if the sequence $\left\{T\left(x_{n}\right)\right\}$ strongly converges to $z$, then $T(\tilde{x})=z$.

In what follows, only the particular case of demiclosedness at zero will be used, which is the particular case when $z=0$. This notion is frequently used in the study of Mann-type iteration.

Recall that the (nearest point or metric) projection from $\mathrm{H}$ onto $\mathrm{C}$, denoted by $\operatorname{proj}_{\mathrm{C}}$, assigns to each $x \in \mathrm{H}$, the unique point $\operatorname{proj}_{\mathrm{C}}(\mathrm{x}) \in \mathrm{C}$ with the property

$$
\left\|x-\operatorname{proj}_{C}(x)\right\|=\inf \{\|x-y\|: y \in C\} .
$$

The metric projection projc of $\mathrm{H}$ onto $\mathrm{C}$ is characterized by

$$
\left\langle x-\operatorname{proj}_{C}(x), y-\operatorname{proj}_{C}(x)\right\rangle \geqslant 0
$$

for all $x \in H, y \in C$.

Definition 2.5. A sequence $\left\{x_{n}\right\}$ is called Fejér-monotone with respect to a given nonempty set $\Omega$ if for every $x \in \Omega$

$$
\left\|x_{n+1}-x\right\| \leqslant\left\|x_{n}-x\right\| \text { for all } n \geqslant 0 .
$$

Next we adopt the following notations:

- $x_{n} \rightarrow x$ means that $x_{n}$ converges weakly to $x$;

- $\omega_{w}\left(x_{n}\right):=\left\{x: \exists x_{n_{j}} \rightarrow x\right\}$ is the weak $\omega$-limit set of the sequence $\left\{x_{n}\right\}$.

Lemma 2.6 ([1]). Let $\Omega$ be a nonempty closed convex subset in $\mathrm{H}$. If the sequence $\left\{x_{n}\right\}$ is Fejér monotone with respect to $\Omega$, then we have the following conclusions:

(i) $x_{n} \rightarrow x^{\dagger} \in \Omega$ iff $\omega_{w}\left(x_{n}\right) \subset \Omega$;

(ii) the sequence $\left\{\operatorname{proj}_{\Omega}\left(x_{n}\right)\right\}$ converges strongly;

(iii) if $x_{n} \rightarrow x^{\dagger} \in \Omega$, then $x^{\dagger}=\lim _{n \rightarrow \infty} \operatorname{proj}_{\Omega}\left(x_{n}\right)$. 


\section{Main results}

In this section, we will consider the split common fixed point problem (1.1) under the following assumptions.

Let $\mathrm{H}_{1}$ and $\mathrm{H}_{2}$ be two real Hilbert spaces. Let $\mathrm{U}: \mathrm{H}_{1} \rightarrow \mathrm{H}_{1}$ and $\mathrm{T}: \mathrm{H}_{2} \rightarrow \mathrm{H}_{2}$ be two demicontractive operators with constants $\beta$ and $\mu$, respectively. Let $A: H_{1} \rightarrow H_{2}$ be a bounded linear operator with its adjoint operator $A^{*}$.

Denote the solution set of problem (1.1) by $\Omega$, that is,

$$
\Omega=\left\{z^{*}: z^{*} \in \operatorname{Fix}(U) \text { and } \operatorname{Az} z^{*} \in \operatorname{Fix}(T)\right\} .
$$

The following lemma plays a key role for solving problem (1.1).

Lemma 3.1. $z^{*}$ solves (1.1) iff $z^{*} \in \operatorname{Fix}\left(U-\tau A^{*}(I-T) A\right)$ for any $\tau>0$.

Proof. If $z^{*}$ solves (1.1), then $z^{*}=U z^{*}$ and $(I-T) A z^{*}=0$. It is obvious that $z^{*} \in \operatorname{Fix}\left(U-\tau A^{*}(I-T) A\right)$ for any $\tau>0$.

To see the converse, let $z^{*} \in \operatorname{Fix}\left(U-\tau A^{*}(I-T) A\right), \forall \tau>0$. Then,

$$
z^{*}=U z^{*}-\tau A^{*}(I-T) A z^{*}
$$

Taking $z \in \Omega$, from (3.1), we obtain

$$
\begin{aligned}
0=\left\langle z^{*}-\mathrm{U} z^{*}+\tau A^{*}(\mathrm{I}-\mathrm{T}) A z^{*}, z^{*}-z\right\rangle & =\left\langle z^{*}-\mathrm{U} z^{*}, z^{*}-z\right\rangle+\tau\left\langle A^{*}(\mathrm{I}-\mathrm{T}) A z^{*}, z^{*}-z\right\rangle \\
& =\left\langle z^{*}-\mathrm{U} z^{*}, z^{*}-z\right\rangle+\tau\left\langle(\mathrm{I}-\mathrm{T}) A z^{*}, A z^{*}-A z\right\rangle .
\end{aligned}
$$

Since $\mathrm{U}$ and $\mathrm{T}$ are demicontractve, from (2.1), we deduce

$$
\left\langle z^{*}-\mathrm{U} z^{*}, z^{*}-z\right\rangle \geqslant \frac{1-\beta}{2}\left\|z^{*}-\mathrm{U} z^{*}\right\|^{2},
$$

and

$$
\left\langle(I-T) A z^{*}, A z^{*}-A z\right\rangle \geqslant \frac{1-\mu}{2}\left\|(I-T) A z^{*}\right\|^{2} .
$$

Hence,

$$
0=\left\langle z^{*}-U z^{*}+\tau A^{*}(I-T) A z^{*}, z^{*}-z\right\rangle \geqslant \frac{1-\beta}{2}\left\|z^{*}-U z^{*}\right\|^{2}+\frac{1-\mu}{2} \tau\left\|(I-T) A z^{*}\right\|^{2} .
$$

Thus, $z^{*} \in \operatorname{Fix}(\mathrm{U})$ and $A z^{*} \in \operatorname{Fix}(T)$ for $\tau>0$ and $\beta, \mu \in[0,1)$. Therefore, $z^{*}$ solves problem (1.1). The proof is completed.

By using Lemma 3.1, we can construct the following Mann's algorithm for solving problem (1.1).

Algorithm 3.2. Initialization: let $x_{0}$ be arbitrary.

Iterative step: for $n \geqslant 0$, given the current iterate $x_{n}$, calculate the next iterate $x_{n+1}$ by the following Mann's form

$$
x_{n+1}=(1-\gamma) x_{n}+\gamma\left[U x_{n}-\tau A^{*}(I-T) A x_{n}\right], n \geqslant 0,
$$

where $\gamma \in\left(0, \frac{1-\beta}{2}\right)$ is a constant.

Theorem 3.3. Assume that $\mathrm{I}-\mathrm{U}$ and $\mathrm{I}-\mathrm{T}$ are demiclosed at zero. If $\Omega \neq \emptyset$, then the sequence $\left\{\mathrm{x}_{\mathrm{n}}\right\}$ generated by (3.2) converges weakly to a solution $z^{*}\left(=\lim _{n \rightarrow \infty} \operatorname{proj}_{\Omega}\left(x_{n}\right)\right)$ of problem (1.1) provided $\tau \in\left(0, \frac{1-\mu}{2 \gamma\|A\|^{2}}\right)$. 
Proof. Firstly, we show that the sequence $\left\{x_{n}\right\}$ is Fejér-monotone with respect to $\Omega$. Taking any $z \in \Omega$, from (2.1), we have

$$
\begin{aligned}
\left\langle x_{n}-\mathrm{U} x_{n}+\tau A^{*}(I-T) A x_{n}, x_{n}-z\right\rangle & =\left\langle x_{n}-U x_{n}, x_{n}-z\right\rangle+\tau\left\langle A^{*}(I-T) A x_{n}, x_{n}-z\right\rangle \\
& =\left\langle x_{n}-U x_{n}, x_{n}-z\right\rangle+\tau\left\langle(I-T) A x_{n}, A x_{n}-A z\right\rangle \\
& \geqslant \frac{1-\beta}{2}\left\|x_{n}-U x_{n}\right\|^{2}+\frac{1-\mu}{2} \tau\left\|(I-T) A x_{n}\right\|^{2} .
\end{aligned}
$$

By (3.2), we get

$$
\begin{aligned}
\left\|x_{n+1}-z\right\|^{2}= & \left\|(1-\gamma) x_{n}+\gamma\left[\mathrm{U} x_{n}-\tau A^{*}(I-T) A x_{n}\right]-z\right\|^{2} \\
= & \left\|x_{n}-z-\gamma\left[x_{n}-U x_{n}+\tau A^{*}(I-T) A x_{n}\right]\right\|^{2} \\
= & \mid x_{n}-z\left\|^{2}-2 \gamma\left\langle x_{n}-U x_{n}+\tau A^{*}(I-T) A x_{n}, x_{n}-z\right\rangle+\gamma^{2}\right\| x_{n}-U x_{n}+\tau A^{*}(I-T) A x_{n} \|^{2} \\
\leqslant & \mid x_{n}-z \|^{2}-2 \gamma\left\langle x_{n}-U x_{n}+\tau A^{*}(I-T) A x_{n}, x_{n}-z\right\rangle+\gamma^{2}\left[\left\|x_{n}-U x_{n}\right\|+\left\|\tau A^{*}(I-T) A x_{n}\right\|\right]^{2} \\
\leqslant & \mid x_{n}-z \|^{2}-2 \gamma\left\langle x_{n}-U x_{n}+\tau A^{*}(I-T) A x_{n}, x_{n}-z\right\rangle \\
& +\gamma^{2}\left[2\left\|x_{n}-U x_{n}\right\|^{2}+2 \tau^{2}\|A\|^{2}\left\|(I-T) A x_{n}\right\|^{2}\right] .
\end{aligned}
$$

This together with (3.3) implies that

$$
\begin{aligned}
\left\|x_{n+1}-z\right\|^{2} \leqslant & \left\|x_{n}-z\right\|^{2}-\gamma\left[(1-\beta)\left\|x_{n}-U x_{n}\right\|^{2}+(1-\mu) \tau\left\|(I-T) A x_{n}\right\|^{2}\right] \\
& +\gamma^{2}\left[2\left\|x_{n}-U x_{n}\right\|^{2}+2 \tau^{2}\|A\|^{2}\left\|(I-T) A x_{n}\right\|^{2}\right] \\
= & \left\|x_{n}-z\right\|^{2}-\gamma(1-\beta-2 \gamma)\left\|x_{n}-U x_{n}\right\|^{2}-\gamma \tau\left(1-\mu-2 \gamma \tau\|A\|^{2}\right)\left\|(I-T) A x_{n}\right\|^{2} .
\end{aligned}
$$

By virtue of (3.4), we deduce that the sequence $\left\{x_{n}\right\}$ is Fejér monotone due to $\gamma \in\left(0, \frac{1-\beta}{2}\right)$ and $\tau \in$ $\left(0, \frac{1-\mu}{2 \gamma\|A\|^{2}}\right)$.

Next, we show that every weak cluster point of the sequence $\left\{x_{n}\right\}$ belongs to the solution set of problem (1.1), i.e., $\omega_{w}\left(x_{n}\right) \subset \Omega$.

From the Fejér-monotonicity of $\left\{x_{n}\right\}$ it follows that the sequence $\left\{x_{n}\right\}$ is bounded. Further, from (3.4), we obtain

$$
\gamma(1-\beta-2 \gamma)\left\|x_{n}-U x_{n}\right\|^{2}+\gamma \tau\left(1-\mu-2 \gamma \tau\|A\|^{2}\right)\left\|(I-T) A x_{n}\right\|^{2} \leqslant\left\|x_{n}-z\right\|^{2}-\left\|x_{n+1}-z\right\|^{2} .
$$

An induction induces that

$$
\begin{gathered}
\gamma(1-\beta-2 \gamma) \sum_{i=1}^{n}\left\|x_{i}-U x_{i}\right\|^{2}+\gamma \tau\left(1-\mu-2 \gamma \tau\|A\|^{2}\right) \sum_{i=1}^{n}\left\|(I-T) A x_{i}\right\|^{2} \\
\leqslant\left\|x_{0}-z\right\|^{2}-\left\|x_{n+1}-z\right\|^{2} \\
\leqslant\left\|x_{0}-z\right\|^{2} .
\end{gathered}
$$

Therefore,

$$
\gamma(1-\beta-2 \gamma) \sum_{i=1}^{\infty}\left\|x_{i}-U x_{i}\right\|^{2}+\gamma \tau\left(1-\mu-2 \gamma \tau\|A\|^{2}\right) \sum_{i=1}^{\infty}\left\|(I-T) A x_{i}\right\|^{2}<\infty,
$$

which implies that

$$
\lim _{n \rightarrow \infty}\left\|x_{n}-U x_{n}\right\|=0 \text { and } \lim _{n \rightarrow \infty}\left\|(I-T) A x_{n}\right\|=0 .
$$

By the demiclosedness (at zero) of $\mathrm{I}-\mathrm{U}$ and $\mathrm{I}-\mathrm{T}$, we deduce immediately $\omega_{w}\left(x_{n}\right) \subset \Omega$. To this end, the conditions of Lemma 2.6 are all satisfied. Consequently, $x_{n} \rightarrow z^{*}=\lim _{n \rightarrow \infty} \operatorname{proj}_{\Omega}\left(x_{n}\right)$. The proof is completed. 
Remark 3.4. Note that (3.2) can be rewritten as

$$
x_{n+1}=x_{n}-\gamma\left[x_{n}-U x_{n}+\tau A^{*}(I-T) A x_{n}\right], n \geqslant 0,
$$

where $\gamma$ is stepsize. In general, $\gamma$ depends on the norm of the transformation $A$. However, we can relax $\gamma$ without relying on the norm of the transformation $A$.

We can apply our results to the split feasibility problem (1.2).

Algorithm 3.5. Initialization: let $x_{0}$ be arbitrary.

Iterative step: for $n \geqslant 0$, given the current iterate $x_{n}$ calculate the next iterate $x_{n+1}$ by the following Mann's form

$$
x_{n+1}=(1-\gamma) x_{n}+\gamma\left[\operatorname{proj}_{C} x_{n}-\tau A^{*}\left(I-\operatorname{proj}_{Q}\right) A x_{n}\right], n \geqslant 0,
$$

where $\gamma \in\left(0, \frac{1-\beta}{2}\right)$ is a constant.

Theorem 3.6. Assume that the problem (1.2) is consistent (i.e., its solution set is nonempty). Then the sequence $\left\{x_{n}\right\}$ generated by (3.5) converges weakly to a solution of the split feasibility problem (1.2) provided $\tau \in\left(0, \frac{1-\mu}{2 \gamma\|A\|^{2}}\right)$.

\section{Acknowledgment}

Yeong-Cheng Liou was supported in part by the grand form Kaohsiung Medical University Research Foundation (KMU-Q106005) and MOST 106-2923-E-039-001-MY3.

\section{References}

[1] H. H. Bauschke, J. M. Borwein, On projection algorithms for solving convex feasibility problems, SIAM Rev., 38 (1996), 367-426. 2.6

[2] F. E. Browder, W. V. Petryshyn, Construction of fixed points of nonlinear mappings in Hilbert space, J. Math. Anal. Appl., 20 (1967), 197-228. 1

[3] C. Byrne, A unified treatment of some iterative algorithms in signal processing and image reconstruction, Inverse Problems, 20 (2004), 103-120. 1.1

[4] L.-C. Ceng, Q. H. Ansari, J.-C. Yao, An extragradient method for solving split feasibility and fixed point problems, Comput. Math. Appl., 64 (2012), 633-642. 1.1

[5] Y. Censor, A. Segal, The split common fixed point problem for directed operators, J. Convex Anal., 16 (2009), 587-600. 1

[6] Z.-H. He, W.-S. Du, On hybrid split problem and its nonlinear algorithms, Fixed Point Theory Appl., 2013 (2013), 20 pages. 1

[7] Ş. Măruşter, C. Popirlan, On the Mann-type iteration and the convex feasibility problem, J. Comput. Appl. Math., 212 (2008), 390-396. 2.3

[8] A. Moudafi, The split common fixed-point problem for demicontractive mappings, Inverse Problems, 26 (2010), 6 pages. 1

[9] F. Wang, Solving the split common fixed point problem by a fixed-point algorithm, J. Nonlinear Sci. Appl., (In press). 1, 1

[10] F.-H. Wang, H.-K. Xu, Cyclic algorithms for split feasibility problems in Hilbert spaces, Nonlinear Anal., 74 (2011), 4105-4111. 1.1

[11] R. Wittmann, Approximation of fixed points of nonexpansive mappings, Arch. Math. (Basel), 58 (1992), 486-491. 1

[12] H.-K. Xu, Viscosity approximation methods for nonexpansive mappings, J. Math. Anal. Appl., 298 (2004), 279-291. 1

[13] H.-K. Xu, Iterative methods for the split feasibility problem in infinite-dimensional Hilbert spaces, Inverse Problems, 26 (2010), 17 pages. 1.1

[14] Y.-H. Yao, R. P. Agarwal, M. Postolache, Y.-C. Liou, Algorithms with strong convergence for the split common solution of the feasibility problem and fixed point problem, Fixed Point Theory Appl., 2014 (2014), 14 pages. 1.1, 1

[15] Y.-H. Yao, R.-D. Chen, J.-C. Yao, Strong convergence and certain control conditions for modified Mann iteration, Nonlinear Anal., 68 (2008), 1687-1693. 1

[16] Y.-H. Yao, W. Jigang, Y.-C. Liou, Regularized methods for the split feasibility problem, Abstr. Appl. Anal., 2012 (2012), 13 pages. 1.1

[17] Y.-H. Yao, L.-M. Leng, M. Postolache, X.-X. Zheng, Mann-type iteration method for solving the split common fixed point problem, J. Nonlinear Convex Anal., (In press). 1

[18] Y.-H. Yao, Y.-C. Liou, S. M. Kang, Approach to common elements of variational inequality problems and fixed point problems via a relaxed extragradient method, Comput. Math. Appl., 59 (2010), 3472-3480. 1 
[19] Y.-H. Yao, Y.-C. Liou, J.-C. Yao, Split common fixed point problem for two quasi-pseudo-contractive operators and its algorithm construction, Fixed Point Theory Appl., 2015 (2015), 19 pages. 1

[20] Y.-H. Yao, M. Postolache, Y.-C. Liou, Strong convergence of a self-adaptive method for the split feasibility problem, Fixed Point Theory Appl., 2013 (2013), 12 pages. 1.1

[21] Y.-H. Yao, N. Shahzad, Y.-C. Liou, Modified semi-implicit midpoint rule for nonexpansive mappings, Fixed Point Theory Appl., 2015 (2015), 15 pages. 1

[22] H. Zegeye, N. Shahzad, Y.-H. Yao, Minimum-norm solution of variational inequality and fixed point problem in Banach spaces, Optimization, 64 (2015), 453-471. 1 